\title{
Aggregatibacter actinomycetemcomitans serotype prevalence and antibiotic resistance in a UK population with periodontitis
}

Chaido Akrivopoulou a , Ingrid M. Green ${ }^{b, c, *}$, Nikolaos Donos ${ }^{a, d}$, Sean P. Nair c, Derren Ready c,e,1

a Periodontology Unit, UCL Eastman Dental Institute, 256 Gray's Inn Road, London WC1X 8LD, UK

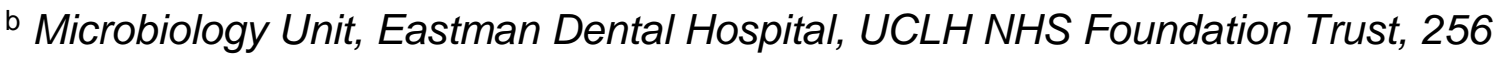

Gray's Inn Road, London WC1X 8LD, UK

c Department of Microbial Diseases, University College London, Eastman Dental Institute, 256 Gray's Inn Road, London WC1X 8LD, UK

'Centre for Oral Clinical Research, Institute of Dentistry, Barts and The London

School of Medicine and Dentistry, Queen Mary University of London, Turner Street, London E1 2AD, UK

e Public Health England, Colindale, London NW9 5EQ, UK

ARTICLE INFO

Article history:

Received 27 January 2017

Accepted 14 March 2017

Keywords:

Periodontitis

Aggregatibacter actinomycetemcomitans 
Antibiotic susceptibility

Antibiotic resistance

Serotype

United Kingdom

* Corresponding author. Present address: Microbiology Unit, Eastman Dental Hospital, UCLH NHS Foundation Trust, 256 Gray's Inn Road, London WC1X 8LD, UK. Tel.: +442034561037.

E-mail address: i.green@ucl.ac.uk (I.M. Green).

${ }^{1}$ Current address: Gastrointestinal Bacteria Reference Unit, National Infection Service, 61 Colindale Avenue, London NW9 5EQ, UK. 


\section{ABSTRACT}

Objectives: Aggregatibacter actinomycetemcomitans is a recognised pathogen involved in aggressive periodontitis. Seven serotypes of $A$. actinomycetemcomitans exist with a range of virulence and distribution dependent on ethnicity and geography. The ability of $A$. actinomycetemcomitans to invade soft tissue can necessitate the use of systemic antibiotics for treatment, however variations in its antibiotic susceptibility exist dependent on geographical location.

Methods: Serotypes of $A$. actinomycetemcomitans isolates from a UK cohort of 50 patients with aggressive periodontitis were determined by PCR. Resistance of the isolates to eight antibiotics [penicillin ( $1 \mathrm{U})$, amoxicillin $(2 \mu \mathrm{g})$, amoxicillin/clavulanic acid $(30 \mu \mathrm{g})$, metronidazole $(5 \mu \mathrm{g})$, clindamycin $(2 \mu \mathrm{g})$, tetracycline $(10 \mu \mathrm{g})$, ciprofloxacin $(5 \mu \mathrm{g})$ and ceftazidime $(30 \mu \mathrm{g})]$ were determined by disk diffusion according to BSAC guidelines.

Results: Prevalences of serotypes a, c, b, e and mixed serotypes were $48 \%, 22 \%$, $2 \%, 2 \%$ and $12 \%$, respectively. The serotype of isolates from seven patients (14\%) could not be deduced by PCR. Of the 56 isolates tested, 100\% were resistant to penicillin and metronidazole, $87.5 \%$ to clindamycin, $83.9 \%$ to amoxicillin and $76.8 \%$ to ceftazidime. Low rates of resistance to tetracycline (8.9\% resistant) and amoxicillin/clavulanic acid (14.3\% resistant) were observed, whereas no isolates were resistant to ciprofloxacin.

Conclusions: As in a number of publications the suggested treatment of aggressive periodontitis includes the combined use of amoxicillin with metronidazole, these results highlight the need for culture and antimicrobial susceptibility investigations in patients with aggressive periodontitis prior to systemic use of antibiotics concomitantly to periodontal therapy. 


\section{Introduction}

Periodontitis, the most prevalent chronic inflammatory disease in humans [1], compromises the integrity of the tooth-supporting tissues, including the gingivae, periodontal ligament and alveolar bone [2]. Aggregatibacter actinomycetemcomitans is a recognised periodontal pathogen involved in the onset of aggressive periodontitis $[3,4]$. There are seven serotypes of $A$. actinomycetemcomitans (named a-g) classified by differences in surface antigens, with certain serotypes and clones being more pathogenic, e.g. serotype b JP2 clone [5]. The distribution of these serotypes varies depending on geography and/or the patient's ethnic background [6]

Patients suffering from aggressive periodontal disease are initially treated by provision of oral hygiene instructions as well as scaling and root planing together with the concomitant use of systemic antibiotics, more specifically with the combined use of amoxicillin and metronidazole [7-9]. Tetracycline, shown to be effective against $A$. actinomycetemcomitans [10,11], is also frequently used in treating localised aggressive periodontitis. However, large variations in the antibiotic susceptibility profiles of $A$. actinomycetemcomitans exist depending on geographical location [12,13], perhaps reflecting different antibiotic usage patterns. Little is known about the $A$. actinomycetemcomitans serotype prevalence and antibiotic resistance profiles in the UK. This study aimed to determine the prevalence of serotypes and the antibiotic resistance profiles of $A$. actinomycetemcomitans associated with aggressive periodontitis in $50 \mathrm{UK}$ patients. 


\section{Materials and methods}

\subsection{Isolation of bacteria}

Aggregatibacter actinomycetemcomitans clinical isolates from 50 patients who had attended the Periodontology Clinic at the Eastman Dental Hospital (London, UK) with suspected aggressive periodontitis and prior to their routine hospital treatment were analysed in this study. These isolates were obtained from routinely collected diagnostic subgingival plaque samples taken from the patients' four deepest periodontal pockets that had been serially diluted in sterile tryptone-soya broth followed by culture onto tryptone-soya-bacitracin-vancomycin agar [14] and incubation anaerobically for 5-7 days as part of the routine diagnostic service.

\section{2. $P C R$ confirmation and serotyping}

All presumptive $A$. actinomycetemcomitans colonies were counted and subcultured and their identity was confirmed by PCR targeting a specific region found in $A$. actinomycetemcomitans on the 16S RNA gene (see Table 1) [15] using the following conditions: 35 cycles of $95^{\circ} \mathrm{C}$ for $1 \mathrm{~min}\left(10 \mathrm{~min}\right.$ for the first cycle), $61^{\circ} \mathrm{C}$ for $1 \mathrm{~min}$ and $72{ }^{\circ} \mathrm{C}$ for $5 \mathrm{~min}$ (10 $\mathrm{min}$ for the final cycle).

All PCR-confirmed $A$. actinomycetemcomitans isolates were further characterised by deducing their serotype by PCR using specific primers (see Table 1). Multiplex PCR was used to deduce serotypes $b, c$ and $f$, and individual $P C R$ reactions were used for serotypes a, $d$ and e [16]. Serotype $g$ was not tested for. Both reactions used the following PCR conditions: 30 cycles at $95^{\circ} \mathrm{C}$ for $30 \mathrm{~s}$ (10 min for the first cycle), 55 ${ }^{\circ} \mathrm{C}$ for $30 \mathrm{~s}$ and $72{ }^{\circ} \mathrm{C}$ for $30 \mathrm{~s}$ (10 min for the last cycle). 
Aggregatibacter actinomycetemcomitans serotype $\mathrm{b}$ isolates were analysed by PCR to determine whether they were of the JP2 clone using in-house primers LeukF and LeukR (see Table 1) under the following conditions: 30 cycles at $95^{\circ} \mathrm{C}$ for $1 \mathrm{~min}(10$ $\min$ for the first cycle), $56{ }^{\circ} \mathrm{C}$ for $1 \mathrm{~min}$ and $72{ }^{\circ} \mathrm{C}$ for $1 \mathrm{~min}(10 \mathrm{~min}$ for the last cycle).

Genomic DNA extracted from pure cultures of reference $A$. actinomycetemcomitans serotypes a-e (HK 929, SUNY 465, HK 914, HK 928 and HK 972, respectively), JP2 [SUNY 465 (JP2) and SUNY 462 (non-JP2)] and serotype f (clinical isolate courtesy of Mogens Kilian, University of Aarhus, Aarhus, Denmark) strains were used as controls.

\subsection{Antibiotic sensitivity and interpretation}

Antibiotic sensitivity was determined by disk diffusion on Iso-Sensitest agar plates (Oxoid Ltd., Basingstoke, UK) supplemented with $5 \%$ defibrinated horse blood (E\&O Laboratories Ltd., Bonnybridge, UK) according to British Society of Antimicrobial Chemotherapy (BSAC) guidelines [17] using the direct inoculation method. Eight antibiotic disks [penicillin (1 U), amoxicillin $(2 \mu \mathrm{g})$, amoxicillin/clavulanic acid (AMC) (30 $\mu \mathrm{g})$, metronidazole $(5 \mu \mathrm{g})$, clindamycin $(2 \mu \mathrm{g})$, tetracycline $(10 \mu \mathrm{g})$, ciprofloxacin $(5 \mu \mathrm{g})$ and ceftazidime $(30 \mu \mathrm{g})$; all from Oxoid Ltd.] were placed onto the surface of the agar plates within 15 min of inoculation and were incubated in air supplemented with $5 \%$ carbon dioxide for 2 days. Metronidazole sensitivity was also tested on fastidious anaerobic agar (FAA) plates (Lab M Ltd., Heywood, UK) supplemented with $5 \%$ defibrinated horse blood (E\&O Laboratories Ltd., Bonnybridge, UK) incubated for 2 days at $37^{\circ} \mathrm{C}$ under anaerobic conditions. Quality control strains 
(Staphylococcus aureus NCTC 6571, Escherichia coli NCTC 12241, Haemophilus influenzae NCTC 11931 and Bacteroides fragilis NCTC 9343; Public Health England, London, UK) were tested simultaneously and their zone diameters were determined to ensure they were within acceptable ranges before interpreting tests. Measured zone diameters were interpreted as being susceptible (S), intermediate-susceptible (I) and resistant (R) according to BSAC [17]. For A. actinomycetemcomitans, the interpretive criteria for the HACEK group were applied for amoxicillin, AMC, ceftazidime, ciprofloxacin and tetracycline. Pasteurella multocida criteria were used for penicillin. No interpretive criteria exist for clindamycin and metronidazole, therefore the interpretive criteria for anaerobes ( $B$. fragilis) were applied as used by Kulik et al. [18].

\subsection{Statistical analysis}

Any differences in the number of antibiotic-resistant $A$. actinomycetemcomitans isolates between the different serotypes was assessed using the $\chi^{2}$ test. Data were analysed using SPSS software version 14.0 , and the $5 \%$ level of statistical significance was used throughout the analyses.

\section{Results}

\subsection{Serotype prevalence}

The median total viable count of the subgingival plaque microbiota was $9.18 \times 10^{6}$ $\mathrm{CFU} / \mathrm{mL}$ (range $6.4 \times 10^{3}$ to $2.5 \times 10^{8} \mathrm{CFU} / \mathrm{mL}$ ) and the median viable count of $A$. actinomycetemcomitans was $5.0 \times 10^{4} \mathrm{CFU} / \mathrm{mL}$ (range $5.5 \times 10^{1} \mathrm{CFU} / \mathrm{mL}$ to $6.6 \times$ 
$\left.10^{6} \mathrm{CFU} / \mathrm{mL}\right)$. The proportion of the cultivable plaque microbiota that comprised $A$. actinomycetemcomitans ranged from $0.001 \%$ in one subject to a maximum of $81.25 \%$ (median $0.63 \%$ ). Of the 50 patients, 24 (48\%) harboured $A$. actinomycetemcomitans serotype a, $11(22 \%)$ serotype c, $1(2 \%)$ serotype b and 1 (2\%) serotype e. Mixed serotype profiles were observed in six of the patients (12\%), five of which were a combination of serotypes a and $c$ and one subject harboured a combination of serotypes $b$ and c. Seven patients (14\%) carried untypeable isolates as no product was amplified with the PCR primer sets. The serotype b isolate was not a JP2 clone as no deletion in the promoter region was detected by PCR analysis of the leukotoxin gene.

\subsection{Antibiotic susceptibility}

All 56 A. actinomycetemcomitans isolates tested were resistant to metronidazole (both in $\mathrm{CO}_{2}$ and anaerobic conditions) and penicillin. Of the 56 isolates, 49 (87.5\%), were resistant to clindamycin, 47 (83.9\%) to amoxicillin and $43(76.8 \%)$ to ceftazidime. Most of the isolates were susceptible to tetracycline, with only $5(8.9 \%)$ of 56 isolates being resistant. Moreover, $8(14.3 \%)$ of the $56 A$. actinomycetemcomitans isolates were resistant to AMC. All of the $A$. actinomycetemcomitans isolates (100\%) were susceptible to ciprofloxacin (Table 2$)$.

The $\chi^{2}$ analysis of the antibiotic resistance profiles of the two $A$. actinomycetemcomitans serotypes found to be most prevalent in this study (serotypes a, $n=29$; and serotype c, $n=17$ ) only demonstrated a significant difference when comparing the number isolates exhibiting resistance to amoxicillin $(P=0.036)$, with $17(100 \%)$ serotype $\mathrm{c}$ isolates resistant to this antibiotic compared 
with $22(75.9 \%)$ of the serotype a isolates demonstrating resistance to this antibiotic (Table 2; Supplementary Table S1).

\section{Discussion}

Serotype a was mostly frequently identified (48\% of patients) in a UK cohort of patients with aggressive periodontitis, followed by serotype c (22\%). Serotypes b and e were recovered from single patient samples. In contrast, in Brazilian, US and Korean samples, serotype c was the most prevalent [52.9\% $(n=85), 42 \%(n=21)$ and $61.9 \%(n=21)$, respectively] [19-21], whereas serotype b [33.3\% $(n=24)]$ was the most common serotype detected in German isolates [21]. In 6 (12\%) of the 50 patients in the current study, two serotypes were detected, similar to the findings of van der Reijden et al. (12.2\%) and Roman-Torres et al. (9.3\%) [19,22]. In the study by van der Reijden, the prevalence of $A$. actinomycetemcomitans serotypes shifted over time [22].

The $b$ serotype isolated in the current study was not a JP2 clone, which concurs with the finding that this highly toxic JP2 clone is predominately recovered in North African [5] rather than in European populations.

\subsection{Antibiotic susceptibilities and resistance}

This study also tested the susceptibility of $A$. actinomycetemcomitans to antibiotics either commonly or infrequently used to treat periodontal disease. 


\subsubsection{Tetracycline}

Tetracycline was found to be one of the most effective antibiotic against the isolates in this study, with 51 (91.1\%) of 56 isolates being susceptible, slightly lower than found with French (96\%; $n=50)$ [23], Swiss $(99.2 \% ; n=125)$ [18], Dutch and Spanish isolates (100\%; $n=18$ and $n=10$, respectively) [24]. In contrast, high tetracycline concentrations were required to kill $90 \%\left(\mathrm{MIC}_{90}=4 \mathrm{mg} / \mathrm{L}\right)$ of Japanese isolates $(n=11)$ [25].

\subsubsection{Clindamycin}

Clindamycin resistance was noted in $87.5 \%$ of the 56 isolates, in keeping with high resistance rates in the USA (93.8\%; $n=81)$ [26], Colombia (83.33\%; $n=18)$ [13] and Switzerland (88\%; $n=125)$ [18]. However, Van Winkelhoff et al. reported lower clindamycin resistance rates for Dutch (22\%) and Spanish (30\%) isolates [24].

\subsubsection{Ceftazidime}

Only $13(23.2 \%)$ of the 56 A. actinomycetemcomitans isolates tested were susceptible to ceftazidime, a third-generation cephalosporin frequently used as a first-line antibiotic in treating $A$. actinomycetemcomitans-associated infective endocarditis [27]. These results contrast with a study of HACEK organisms [28] that found third-generation cephalosporins to be most effective, with ceftriaxone having an MIC range of $0.006-0.023 \mathrm{mg} / \mathrm{L}(n=5)$. 


\subsubsection{Penicillin}

Although penicillin is commonly used against micro-organisms of the HACEK group, no $A$. actinomycetemcomitans isolates were susceptible to penicillin in this study, in line with the susceptibility rates in studies conducted in Germany (6.2\%) [29] and Switzerland (12\%) [18]. A French study found higher susceptible rates $(60 \% ; n=50)$ [23], similar to those found in Dutch $(55.6 \% ; n=18)$ and Spanish $(60.6 \% ; n=10)$ isolates.

\subsubsection{Amoxicillin}

The majority (83.9\%) of the A. actinomycetemcomitans isolates in the present study were resistant to amoxicillin, similar to rates in a Columbian study $(77.7 \% ; n=18)$ [13]. A number of studies have reported high susceptibility rates (93.9-100\%) of $A$. actinomycetemcomitans isolates to amoxicillin $[23,24,26]$, however breakpoint concentrations in these studies were considerably higher than the BSAC definition of resistance in HACEK organisms [30], similar to an issue found in studies that showed $33.3 \%$ and $25 \%$ resistance rates $[24,29]$ and therefore these results are not comparable.

\subsubsection{Amoxicillin/clavulanic acid (AMC)}

The combination AMC reduced the proportion of resistance from $83.9 \%$ for amoxicillin alone to $14.3 \%$ in isolates in the current study. In a Columbian study, similar reductions occurred ( $77.77 \%$ to $0 \%)$ [13]. Results from a Spanish group reported a resistance decrease from $33.3 \%$ to $10 \%$ with the addition of clavulanic acid [24]. 


\subsubsection{Metronidazole}

Metronidazole was one of the least effective antibiotics used in this study $(100 \%$ resistance), results that are corroborated by other studies $[13,23,25,31]$. Lower resistance rates of $20.8 \%$ [18] and $37.5 \%$ [29] have been reported when performed under anaerobic conditions [32]. There was no increase in zone diameter when incubated anaerobically, implying that the variance in resistance rates in the abovementioned studies was probably due to geographical distribution or the breakpoint chosen as, e.g., the breakpoint chosen by Eick et al. was $\geq 32 \mathrm{mg} / \mathrm{L}$ [29].

\subsubsection{Ciprofloxacin}

All 56 A. actinomycetemcomitans isolates (100\%) were shown to be susceptible to ciprofloxacin, in line with the high susceptibility rates in isolates found in Germany [29,31], France [23], Finland [33] and Japan [25].

\subsection{Serotype-specific resistance}

The only significant difference in the proportion of antibiotic-resistant serotype a and c isolates was found in susceptibility to amoxicillin, with $24.1 \%$ and $0 \%$ of serotype a and $\mathrm{c}$ isolates sensitive to this antibiotic, respectively $(P=0.036)$. Pajukanta et al. found only small differences in the MIC of ceftazidime between different serotypes ( $n$ $=80)$ [33], whereas a smaller study by Ihalin et al. $(n=12)$ reported substantial differences in sensitives between different serotypes [34], although these results should be treated with caution owing to the sample size. 
The large variation in antibiotic susceptibility rates between studies may be attributed to different levels of antibiotic consumption [24] as seen in data published in 2013 by the European Surveillance of Antimicrobial Consumption (ESAC) [35]. Total outpatient antibiotic use in 2013 varied by a factor of almost three between countries. Local observation data are decisive and should be utilised to direct clinical supervision, to modernise treatment procedures, to instruct prescribers and to conduct infection control policies [36].

To determine which antibiotic should be selected as an adjunct in $A$. actinomycetemcomitans-associated periodontitis therapy, most clinical laboratories, use the disk diffusion method. This method does not allow determination of the MIC or consider the influence of periodontal biofilms on susceptibility [37-39]. Other considerations include the ability of $A$. actinomycetemcomitans to invade and grow in human cells [40] and the ability to achieve a therapeutic level of the antibiotic in gingival crevicular fluid and saliva. Fluoroquinolones, which have higher bioavailability levels in saliva than in plasma [31] coupled with the high susceptibility rates of $A$. actinomycetemcomitans to this antibiotic class observed in this study, could make them an option for treating unresponsive $A$. actinomycetemcomitans periodontitis.

\section{Conclusion}

This is the first study examining the serotype prevalence and $A$. actinomycetemcomitans antibiotic resistance in a UK population of periodontitis patients. Caution is required prior to prescription of antibiotics for the treatment of 
periodontal disease and, if necessary, microbial testing should be carried out before treatment commences.

Acknowledgments: Thanks to Mogens Kilian (Institute of Medical Microbiology and Immunology, University of Aarhus, Aarhus, Denmark) for kindly providing the serotype $\mathrm{f}$ clinical isolate.

Funding: This work was undertaken at University College London Hospitals/University College Hospital (UCLH/UCL), which received a proportion of funding from the Department of Health's National Institute for Health Research (NIHR) Biomedical Research Centres Funding Scheme, UK.

Competing interests: None declared.

Ethical approval: Not required. 


\section{References}

[1] Richards D. Oral diseases affect some 3.9 billion people. Evid Based Dent 2013;14:35.

[2] Hajishengallis G. Periodontitis: from microbial immune subversion to systemic inflammation. Nat Rev Immunol 2015;15:30-44.

[3] Genco R, Kornman K, Williams R, Offenbacher S, Zambon JJ, Listgarten M, et al. Consensus report. Periodontal diseases: pathogenesis and microbial factors. Ann Periodontol 1996;1:926-32.

[4] Henderson B, Ward JM, Ready D. Aggregatibacter (Actinobacillus) actinomycetemcomitans: a triple $A^{*}$ periodontopathogen? Periodontol 2000 2010;54:78-105.

[5] Haubek D, Ennibi OK, Poulsen K, Væth M, Poulsen S, Kilian M. Risk of aggressive periodontitis in adolescent carriers of the JP2 clone of Aggregatibacter (Actinobacillus) actinomycetemcomitans in Morocco: a prospective longitudinal cohort study. Lancet 2008;371:237-42.

[6] Rylev M, Kilian M. Prevalence and distribution of principal periodontal pathogens worldwide. J Clin Periodontol 2008;35(8 Suppl):346-61.

[7] Sgolastra F, Petrucci A, Gatto R, Monaco A. Effectiveness of systemic amoxicillin/metronidazole as an adjunctive therapy to full-mouth scaling and root planing in the treatment of aggressive periodontitis: a systematic review and meta-analysis. J Periodontol 2012;83:731-43.

[8] Teughels W, Dhondt R, Dekeyser C, Quirynen M. Treatment of aggressive periodontitis. Periodontol 2000 2014;65:107-33.

[9] Matthews DC. Adjunctive antibiotics in the treatment of generalized aggressive periodontitis. Evid Based Dent 2006;7:67. 
[10] Slots J, Rosling BG. Suppression of the periodontopathic microflora in localized juvenile periodontitis by systemic tetracycline. J Clin Periodontol $1983 ; 10: 465-86$.

[11] Kornman KS, Robertson PB. Clinical and microbiological evaluation of therapy for juvenile periodontitis. J Periodontol 1985;56:443-6.

[12] Veloo AC, Seme K, Raangs E, Rurenga P, Singadji Z, Wekema-Mulder G, et al. Antibiotic susceptibility profiles of oral pathogens. Int J Antimicrob Agents 2012;40:450-4.

[13] Ardila CM, Granada MI, Guzmán IC. Antibiotic resistance of subgingival species in chronic periodontitis patients. J Periodontal Res 2010;45:557-63.

[14] Slots J. Selective medium for isolation of Actinobacillus actinomycetemcomitans. J Clin Microbiol 1982;15:606-9.

[15] Gafan GP, Lucas VS, Roberts GJ, Petrie A, Wilson M, Spratt DA. Prevalence of periodontal pathogens in dental plaque of children. J Clin Microbiol 2004;42:4141-6.

Kaplan JB, Perry MB, MacLean LL, Furgang D, Wilson ME, Fine DH.

Structural and genetic analyses of $O$ polysaccharide from Actinobacillus actinomycetemcomitans serotype f. Infect Immun 2001;69:5375-84.

[17] Howe RA, Andrews JM; BSAC Working Party on Susceptibility Testing. BSAC standardized disc susceptibility testing method (version 11). J Antimicrob Chemother 2012;67:2783-4.

[18] Kulik EM, Lenkeit K, Chenaux S, Meyer J. Antimicrobial susceptibility of periodontopathogenic bacteria. J Antimicrob Chemother 2008;61:1087-91. 
[19] Roman-Torres CV, Aquino DR, Cortelli SC, Franco GC, dos Santos JG, Corraini P, et al. Prevalence and distribution of serotype-specific genotypes of Aggregatibacter actinomycetemcomitans in chronic periodontitis Brazilian subjects. Arch Oral Biol 2010;55:242-8. actinomycetemcomitans serotypes in subgingival plaque from United States subjects. Mol Oral Microbiol 2010;25:207-14.

[21] Kim T-S, Frank P, Eickholz P, Eick S, Kim CK. Serotypes of Aggregatibacter actinomycetemcomitans in patients with different ethnic backgrounds. J Periodontol 2009;80:2020-7.

[22] van der Reijden WA, Bosch-Tijhof CJ, van der Velden U, van Winkelhoff AJ. Java project on periodontal diseases: serotype distribution of Aggregatibacter actinomycetemcomitans and serotype dynamics over an 8year period. J Clin Periodontol 2008;35:487-92.

[23] Madinier IM, Fosse TB, Hitzig C, Charbit Y, Hannoun LR. Resistance profile survey of 50 periodontal strains of Actinobacillus actinomyectomcomitans. J Periodontol 1999;70:888-92.

[24] Van Winkelhoff AJ, Herrera D, Oteo A, Sanz M. Antimicrobial profiles of periodontal pathogens isolated from periodontitis patients in The Netherlands and Spain. J Clin Periodontol 2005;32:893-8.

[25] Miyake Y, Tsuruda K, Okuda K, Widowati, Iwamoto Y, Suginaka H. In vitro activity of tetracyclines, macrolides, quinolones, clindamycin and metronidazole against periodontopathic bacteria. J Periodontal Res 1995;30:290-3. 
[26] Rams TE, Degener JE, van Winkelhoff AJ. Antibiotic resistance in human chronic periodontitis microbiota. J Periodontol 2014;85:160-9.

[27] Paturel L, Casalta JP, Habib G, Nezri M, Raoult D. Actinobacillus actinomycetemcomitans endocarditis. Clin Microbiol Infect 2004;10:98-118.

[28] Kugler KC, Biedenbach DJ, Jones RN. Determination of the antimicrobial activity of 29 clinically important compounds tested against fastidious HACEK group organisms. Diagn Microbiol Infect Dis 1999;34:73-6.

[29] Eick S, Pfister W, Straube E. Antimicrobial susceptibility of anaerobic and capnophilic bacteria isolated from odontogenic abscesses and rapidly progressive periodontitis. Int J Antimicrob Agents 1999;12:41-6.

[30] British Society for Antimicrobial Chemotherapy. BSAC methods for antimicrobial susceptibility testing. Version 14 January 2015. BSAC; 2015. http://bsac.org.uk/wp-content/uploads/2012/02/BSAC-disc-susceptibilitytesting-method-Jan-2015.pdf [accessed 20 June 2017].

[31] Müller H-P, Holderrieth S, Burkhardt U, Höffler U. In vitro antimicrobial susceptibility of oral strains of Actinobacillus actinomycetemcomitans to seven antibiotics. J Clin Periodontol 2002;29:736-42.

[32] Edwards DI. Nitroimidazole drugs_-action and resistance mechanisms. I. Mechanisms of action. J Antimicrob Chemother 1993;31:9-20.

[33] Pajukanta R, Asikainen S, Saarela M, Alaluusua S, Jousimies-Somer H. In vitro antimicrobial susceptibility of different serotypes of Actinobacillus actinomycetemcomitans. Scand J Dent Res 1993;101:299-303.

[34] Ihalin R, Pienihäkkinen K, Lenander M, Tenovuo J, Jousimies-Somer H. Susceptibilities of different Actinobacillus actinomycetemcomitans strains 
to lactoperoxidase-iodide-hydrogen peroxide combination and different antibiotics. Int J Antimicrob Agents 2003;21:434-40.

Weist K, Diaz Högberg L. ECDC publishes 2013 surveillance data on antimicrobial resistance and antimicrobial consumption in Europe. Euro Surveill 2014;19:pii: 20962.

[36] Simonsen GS, Tapsall JW, Allegranzi B, Talbot EA, Lazzari S. The antimicrobial resistance containment and surveillance approach—a public health tool. Bull World Health Organ 2004;82:928-34.

[37] Schaudinn C, Gorur A, Keller D, Sedghizadeh PP, Costerton JW. Periodontitis: an archetypical biofilm disease. J Am Dent Assoc 2009;140:978-86.

[38] Filoche S, Wong L, Sissons CH. Oral biofilms: emerging concepts in microbial ecology. J Dent Res 2010;89:8-18.

[39] Armitage GC. Comparison of the microbiological features of chronic and aggressive periodontitis. Periodontol 2000 2010;53:70-88.

[40] Rudney JD, Chen R, Sedgewick GJ. Intracellular Actinobacillus actinomycetemcomitans and Porphyromonas gingivalis in buccal epithelial cells collected from human subjects. Infect Immun 2001;69:2700-7. 